\title{
GALACTIC Al 1.8 MeV GAMMA-RAY SURVEYS WITH INTEGRAL
}

\author{
WEI WANG \\ National Astronomical Observatories \\ Chinese Academy of Sciences \\ Beijing 100012, China \\ wangwei@bao.ac.cn
}

\begin{abstract}
${ }^{26} \mathrm{Al}$ is a long-life radioactive isotope with a half lifetime of near $1 \mathrm{Myr}$. The origin of Galactic ${ }^{26} \mathrm{Al}$ is dominated by massive stars and their core-collapse supernovae. Detections of $1809 \mathrm{keV}$ emission from ${ }^{26} \mathrm{Al}$ provide direct evidence that nucleosynthesis is ongoing in the Galaxy. The gamma-ray line shapes reflect the dynamics of the ejected isotopes in the interstellar medium and then probe properties of ISM and Galactic rotation effect. Gamma-ray emissions of ${ }^{26} \mathrm{Al}$ in the Galaxy are studied with the high spectral resolution INTEGRAL spectrometer (SPI). We carry out the first spectral survey of ${ }^{26} \mathrm{Al}$ gamma-ray line emission along the Galactic plane. The ${ }^{26} \mathrm{Al}$ line energy shifts reflect the large-scale Galactic rotation. The ${ }^{26} \mathrm{Al}$ intensity is brighter in the 4 th than in the 1 st quadrant (ratio $\sim 1.3$ ); the ${ }^{26} \mathrm{Al}$ line toward the direction of the Aquila region appears somewhat broadened; a latitudinal scale height of $130_{-70}^{+120} \mathrm{pc}$ for ${ }^{26} \mathrm{Al}$ in the inner Galaxy is determined. Strong ${ }^{26} \mathrm{Al}$ emission signal is detected in the nearby starformation regions Sco-Cen and Cygnus. The ${ }^{26} \mathrm{Al}$ line shapes in star-formation regions provide a clue to constrain the kinematic properties of ISM. In addition, we derive the flux ratio of ${ }^{60} \mathrm{Fe} /{ }^{26} \mathrm{Al} \sim 15 \%$ which can be directly compared with theoretical predictions. More theoretical work on nuclear reactions, massive star evolution models deserves improvements
\end{abstract}

Keywords: Gamma-rays: observations; Galaxy: abundances; nucleosynthesis.

\section{Origin of Galactic ${ }^{26} \mathrm{Al}$}

${ }^{26} \mathrm{Al}$ is an unstable nucleus, produced almost exclusively by proton capture on ${ }^{25} \mathrm{Mg}$ in a sufficiently hot environment, ${ }^{15}$ mainly destroyed by the $\beta^{+}$decay into ${ }^{26} \mathrm{Al}$ since the competing destruction process, i.e., the ${ }^{26} \mathrm{Al}(p, \gamma){ }^{27} \mathrm{Si}$ reaction, becomes efficient for $T>5 \times 10^{7} \mathrm{~K}$, and the end of central the $\mathrm{H}$ burning barely reaches such a temperature. In addition, the ${ }^{26} \mathrm{Al}$ freshly synthesized must be ejected into the interstellar medium before it is destroyed. So its synthesis occurs, essentially in three different specific environments: i.e., the core $\mathrm{H}$ burning, the $\mathrm{C}$ and $\mathrm{Ne}$ convective shells, and the explosive Ne burning. For the hydrostatic nucleosynthesis in the core of stars with convective envelopes, the fresh ${ }^{26} \mathrm{Al}$ requires to be convected away from the hot inner burning region sufficiently fast to prevent destruction, and ejected by strong stellar winds. The present theoretical knowledge of ${ }^{26} \mathrm{Al}$ origin in the Galaxy 


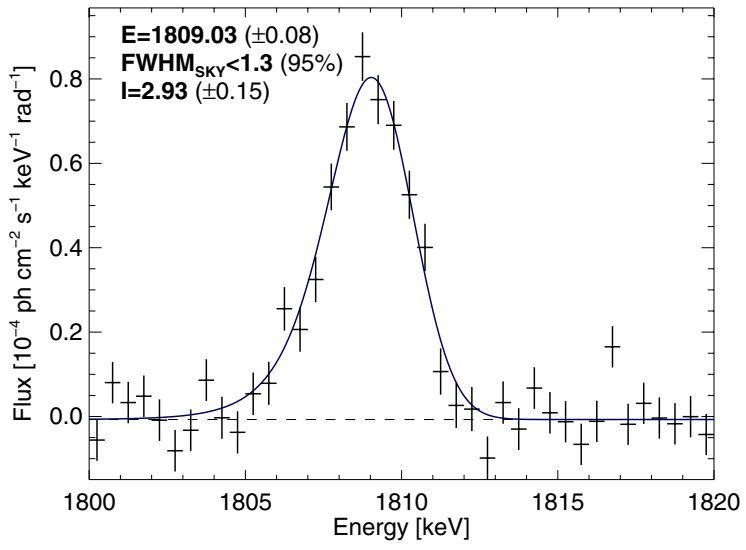

Fig. 1. ${ }^{26} \mathrm{Al}$ spectrum of the inner Galaxy from INTEGRAL/SPI observations.

suggests five possible origins of ${ }^{26} \mathrm{Al}$ : core-collapse supernovae; Wolf-Rayet stars; novae; asymptotic giant branch (AGB) stars; and cosmic-ray nuclear reactions in the interstellar medium.

From the all-sky image survey of $1809 \mathrm{keV}$ emission by COMPTEL, ${ }^{7}$ the ${ }^{26} \mathrm{Al}$ in the interstellar medium is predominantly synthesized in massive stars, through the strong stellar winds of Wolf-Rayet stars and core-collapse supernova explosions. The total ${ }^{26} \mathrm{Al}$ yields due to two processes have been calculated by different work. ${ }^{5,16}$ The average ${ }^{26} \mathrm{Al}$ yields provided by various work range from $(0.3-30) \times 10^{-5} M_{\odot}$ for $10-35 M_{\odot}$ stars, and from $(1-10) \times 10^{-4} M_{\odot}$ for stars above $35 M_{\odot}$

\section{2. ${ }^{26} \mathrm{Al} 1.8 \mathrm{MeV}$ Gamma-Ray Surveys of Galactic Plane}

INTEGRAL is an European (ESA) Gamma-Ray Observatory Satellite Mission for the study of cosmic gamma-ray sources in the $\mathrm{keV}$ to $\mathrm{MeV}$ energy range. The INTEGRAL spectrometer (SPI) can allow for high spectral resolution of $\sim 2.5 \mathrm{keV}$ at $1 \mathrm{MeV}$, suitable for astrophysical studies of individual gamma-ray lines and their shapes. Detailed ${ }^{26} \mathrm{Al}$ spectral surveyes along the Galactic plane will provide new information on ${ }^{26} \mathrm{Al}$ ejecta through the line shapes, e.g., Doppler effect induced from Galactic rotation and dynamics of the ejected ${ }^{26} \mathrm{Al}$ as it propagates in the interstellar medium.

Using more than five years of SPI data, the inner-Galaxy ${ }^{26} \mathrm{Al}$ emission spectrum is shown in Fig. 7. The ${ }^{26} \mathrm{Al}$ line is detected at $\sim 28 \sigma$ significance. ${ }^{14}$ The ${ }^{26} \mathrm{Al}$ gammaray flux from the inner Galaxy turns out as $(2.93 \pm 0.15) \times 10^{-4} \mathrm{ph} \mathrm{cm}^{-2} \mathrm{~s}^{-1} \mathrm{rad}^{-1}$. The measured ${ }^{26} \mathrm{Al}$ flux for the inner Galaxy translates into a Galactic ${ }^{26} \mathrm{Al}$ mass of $(2.6 \pm 0.6) M_{\odot}$ using a scale height of $180 \mathrm{pc},{ }^{14}$ which corresponds to a core-collapse supernova rate $\sim 1.90 \pm 0.95$ in the Galaxy. 

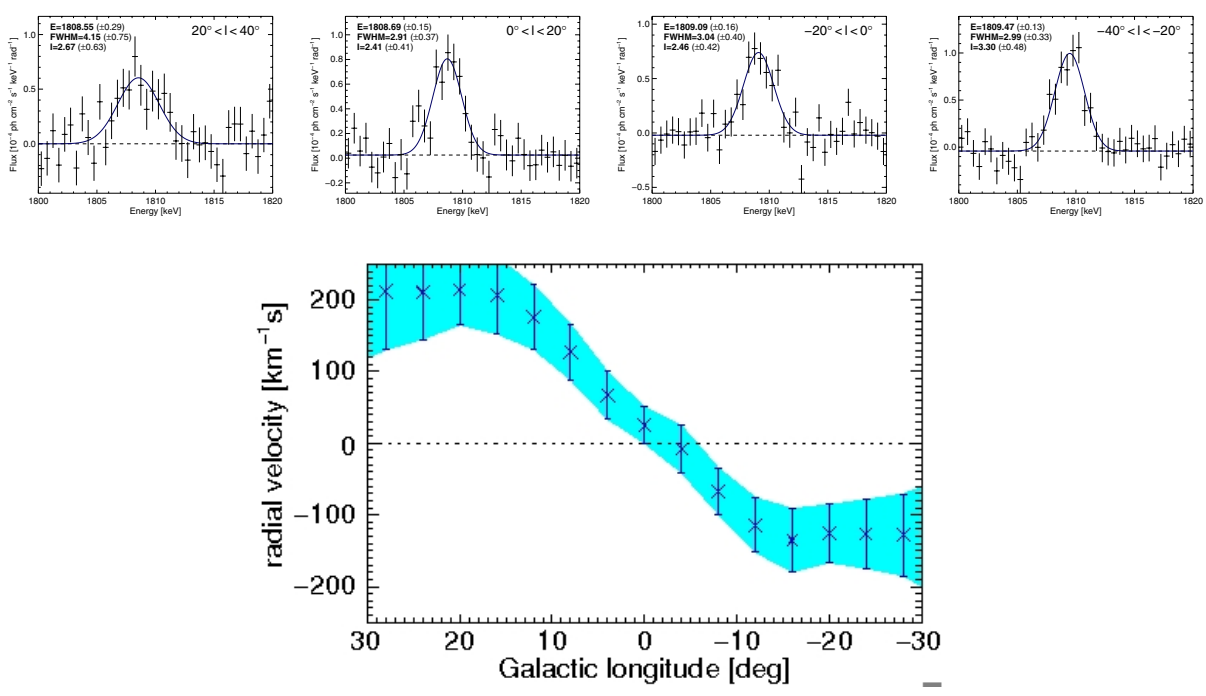

Fig. 2. Top ${ }^{26} \mathrm{Al}$ spectral variations along the Galactic plane; Bottom Radial velocity curve of ${ }^{26} \mathrm{Al}$ ejecta in the stellar medium along Galactic longitudes which shows Doppler shifts of the 1809 $\mathrm{keV}$ gamma-ray line from ${ }^{26} \mathrm{Al}$ due to Galactic rotation.

SPI can also probe the ${ }^{26} \mathrm{Al}$ variations along the Galactic plane due to its imaging properties as a coded-mask telescope. The ${ }^{26} \mathrm{Al}$ emission asymmetry between the two inner Galactic quadrants was detected, with a flux ratio (4th/1st) of $\sim 1.3 \pm$ 0.2. ${ }^{14}$ Fig. 2 (top panel) shows the ${ }^{26} \mathrm{Al}$ spectra with $20^{\circ}$ regions along the Galactic plane. The variability of ${ }^{26} \mathrm{Al}$ intensity along the Galactic plane again is evident. In addition, the ${ }^{26} \mathrm{Al}$ line in the region of $20^{\circ}<l<40^{\circ}$ appears somewhat broadened, with a Gaussian width of FWHM $4.15 \pm 0.75 \mathrm{keV}$. This may hint towards a peculiar ${ }^{26} \mathrm{Al}$ source region towards this direction, which could be associated with the Aquila region.

Galactic differential rotation can result in not only the broadening of ${ }^{26} \mathrm{Al}$ line but also the shifts of the line centroid energy due to the Doppler effect. ${ }^{2,3}$ Galactic rotation would induce the ${ }^{26} \mathrm{Al}$ line centroid energy redshifts in the 1st quadrant and blueshifts in the 4th quadrant. Fig. 2 (bottom panel) presents the radial velocity curve of ${ }^{26} \mathrm{Al}$ ejecta along Galactic longitudes with high resolution. ${ }^{4}$ The measurements show the characteristic wave-like shape of velocity offset versus Galactic longitude due to the large-scale Galactic rotation. This has been observed for molecular and atomic gas in our and other galaxies. But with ${ }^{26} \mathrm{Al}$ gamma-rays, we get unique access to kinematics of the tenuous, diluted, and presumably hot phase of the interstellar medium, as it is expected around the massive stars which eject radioactive ${ }^{26} \mathrm{Al}$ in the Galaxy. We should note that comparing the velocity curves from ${ }^{26} \mathrm{Al}$ line shape measurements along the Galactic plane and other observations and theory found that the hot gas of ${ }^{26} \mathrm{Al}$ ejecta undergoes additional acceleration in the direction of Galactic rotation. 
We also determine the scale height of the Galactic plane in ${ }^{26} \mathrm{Al}$ emission by comparing the fit quality for sets of two different plausible geometrical models for the ${ }^{26} \mathrm{Al}$ source density distribution in the Galaxy (a Glactocentric double-exponential disk model and a spiral-arm structure model based on Ref.9, varying their scale height parameters). A scale height of $130_{-70}^{+120} \mathrm{pc}(1 \sigma)$ for the ${ }^{26} \mathrm{Al}$ emission in the inner Galactic disk is derived.

\section{3. ${ }^{26} \mathrm{Al}$ Line Shapes in Star-Formation Regions}

Detections of ${ }^{26} \mathrm{Al}$ in star-formation regions will strongly support the hypothesis of massive stars and their descendent supernovae being the dominant sources of interstellar ${ }^{26} \mathrm{Al}$. SPI has obtained the ${ }^{26} \mathrm{Al}$ line shapes for two nearby regions: Cygnus and Sco-Cen.

\subsection{Cygnus}

The Cygnus region is one of the most active nearby star forming regions in the Galaxy with a mean age of $\sim 3 \mathrm{Myr}$, which contains numerous massive stars, supernova remnants. The ${ }^{26} \mathrm{Al}$ emission from the Cygnus region was first reported by the COMPTEL imaging observations, but no spectral information. ${ }^{26} \mathrm{Al}$ line shape of Cygnus has been detailedly studied by SPI (Fig. 3 left panel). The line shape appears a narrow line feature with an intrinsic FWHM of $0.9 \pm 0.8 \mathrm{keV}^{11,13}$, with a flux of $(6.0 \pm 1.0) \times 10^{-5} \mathrm{ph} \mathrm{cm}^{-2} \mathrm{~s}^{-1}$. The ${ }^{26} \mathrm{Al}$ decay emission from Cygnus was represented as a $3^{\circ} \times 3^{\circ}$ Gaussian centred on $(l, b)=\left(81^{\circ}, 0.1^{\circ}\right)$, a position consistent with that of the massive Cyg OB2 cluster thought to dominate the energetics and nucleosynthesis of the Cygnus complex. ${ }^{6}$

\subsection{Sco-Cen}

The nearest site of massive star formation, the Scorpius-Centaurus-Lupus region (hereafter the Sco-Cen region), is about $100-150$ pc from the Sun. ${ }^{26} \mathrm{Al}$ gamma-ray
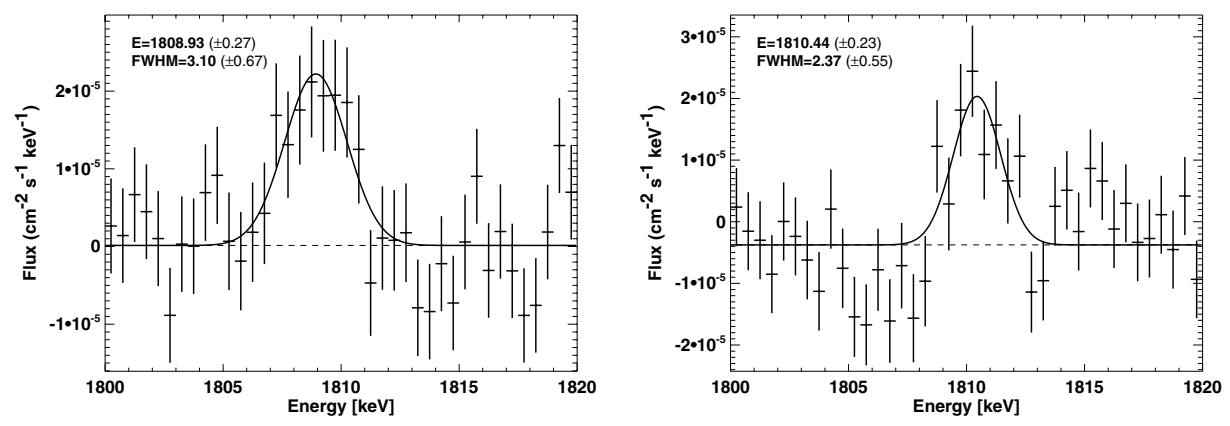

Fig. 3. ${ }^{26} \mathrm{Al}$ spectra of the Cygnus (left) and Sco-Cen (right) regions. 
signal toward Sco-Cen is detected with a significance level of $\sim 5 \sigma .{ }^{1}$ The ${ }^{26} \mathrm{Al}$ line spectrum toward the Sco-Cen region is presented in Fig. 3 (right panel). The observed flux of Sco-Cen is about $6 \times 10^{-5} \mathrm{ph} \mathrm{cm}^{-2} \mathrm{~s}^{-1}$. This ${ }^{26} \mathrm{Al}$ flux corresponds to $1.1 \times 10^{-4} M_{\odot}$ at a distance of $150 \mathrm{pc}$. A typical ${ }^{26} \mathrm{Al}$ yield from a massive star in mass range 8-40 $M_{\odot}$ is about $10^{-4} M_{\odot},{ }^{5,17}$ which is consistent with the derived ${ }^{26} \mathrm{Al}$ mass from observations.

\section{The Ratio of ${ }^{60} \mathrm{Fe} /{ }^{26} \mathrm{Al}$}

${ }^{60} \mathrm{Fe}$ is an unstable nucleus whose terrestrial half-life is $\simeq 1.5 \times 10^{6}$ years. The ${ }^{60} \mathrm{Fe}$ isotope is synthesized in neutron capture reactions from the ${ }^{56} \mathrm{Fe}$ isotope. ${ }^{60} \mathrm{Fe}$ can be produced in massive stars, before or during their final evolution to core collapse supernovae, and Type Ia supernovae. Hence, ${ }^{26} \mathrm{Al}$ and ${ }^{60} \mathrm{Fe}$ would share at least some of the same production sites, i.e. massive stars and supernovae.

Different theoretical models have predicted the ratio of ${ }^{60} \mathrm{Fe} /{ }^{26} \mathrm{Al} \cdot{ }^{10,8,5}$ Gammaray observations could detect these two isotopes and report the flux ratio of ${ }^{60} \mathrm{Fe} /{ }^{26} \mathrm{Al}$ which can be directly compared with theories. Therefore, the measurement of the gamma-ray flux ratio ${ }^{60} \mathrm{Fe} /{ }^{26} \mathrm{Al}$ is important for discussions of the astrophysical origins of the two radioactive isotopes, and the nuclear physics involved in models for their production.

Before SPI, ${ }^{60} \mathrm{Fe}$ signal in the Galaxy is never confirmed by observations. With the SPI data, we detected the diffuse ${ }^{60} \mathrm{Fe}$ emission in the Galaxy with a significance level of $\sim 5 \sigma$ by superposing two gamma-ray lines at $1173 \mathrm{keV}$ and $1332 \mathrm{keV}$ (Fig. 4). The line flux estimated from the combined spectrum is $(4.4 \pm 0.9) \times 10^{-5} \mathrm{ph} \mathrm{cm}^{-2} \mathrm{~s}^{-1} \mathrm{rad}^{-1}$. Then we find a flux ratio of $F\left({ }^{60} \mathrm{Fe}\right) / F\left({ }^{26} \mathrm{Al}\right)=$ $0.148 \pm 0.06 .^{12}$

Theoretical predictions of the ratio of ${ }^{60} \mathrm{Fe} /{ }^{26} \mathrm{Al}$ have undergone some changes in the last ten years. Ref.10 published the first detailed theoretical prediction of

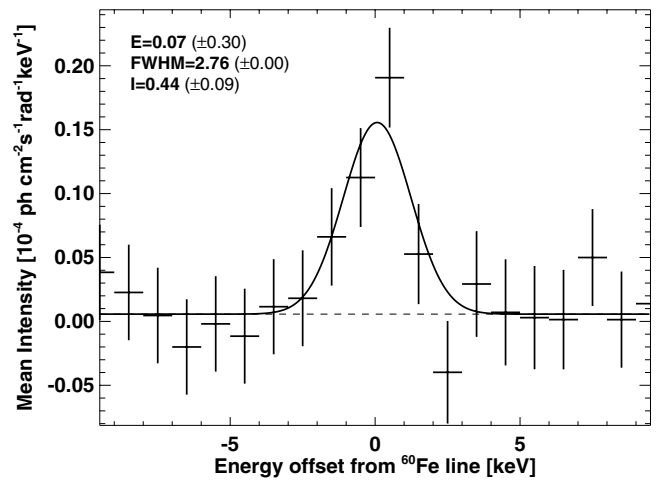

Fig. 4. The combined spectrum of the ${ }^{60} \mathrm{Fe}$ signal in the inner Galaxy by SPI (superimposing two gamma-ray lines at $1773 \mathrm{keV}$ and $1332 \mathrm{keV}$ ). 
a gamma-ray flux ratio $0.16 \pm 0.12$. This prediction would be consistent with our present measurements. Since 2002, theoreticians have improved various aspects of the stellar-evolution models, including improved stellar wind models and the corresponding mass loss effects on stellar structure and evolution, of mixing effects from rotation, and also updated nuclear cross sections in the nucleosynthesis parts of the models. As a result, predicted flux ratios ${ }^{60} \mathrm{Fe} /{ }^{26} \mathrm{Al}$ rather fell into the range $0.8 \pm 0.4 .^{8}$ Such high values would be inconsistent with the SPI result. Recently, new ${ }^{26} \mathrm{Al}$ and ${ }^{60} \mathrm{Fe}$ yield models predict a lower prediction for the ${ }^{60} \mathrm{Fe} /{ }^{26} \mathrm{Al}$ flux ratio of $0.185 \pm 0.0625$, which is again consistent with the observational constraints. ${ }^{5}$

In summary, uncertainties still exist, both in models and measurements of ${ }^{26} \mathrm{Al}$ and ${ }^{60} \mathrm{Fe}$. On the theory side, stellar evolution in late stages is complex, nuclear reactions include neutron capture on unstable Fe isotopes, and explosive nucleosynthesis adds yet another complex ingredient. On the experimental side, the half-lives of ${ }^{26} \mathrm{Al}$ and ${ }^{60} \mathrm{Fe}$, cosmic ray induced ${ }^{60} \mathrm{Co}$ radioactivity in the instrument and spacecraft and the limitations of spatial resolutions and sensitivity are issues reflected in the substantial uncertainties in experimental values.

\section{Acknowledgments}

This work is supported by the NSFC Nos. 10803009, 10833003, 11073030.

\section{References}

1. Diehl, R. et al. A\&A 522 (2010) 51

2. Gehrels, N. and Chen, W. A\&SAS 120 (1996) 331

3. Kretschmer, K. et al.. A\&SA 412 (2003) L47

4. Kretschmer, K. PhD Thesis, Technische Universität München (2011)

5. Limongi, M. and Chieffi, A. ApJ 647 (2006) 483

6. Martin, P. et al. A\&A 506 (2009) 703

7. Plüschke, S. et al. in ESA SP: Exploring the Gamma-Ray Universe ed. Gimenez, A. and Reglero, V. and Winkler, C. 459 (2001) 55

8. Prantzos, N. A $\mathscr{E} A 420$ (2004) 1033

9. Taylor, J. H. and Cordes, J. M. ApJ 411 (1993) 674

10. Timmes, F. X. et al. ApJ 449 (1995) 204

11. Wang, W. PhD Thesis, Technische Universität München (2007)

12. Wang, W. et al. A\&A 469 (2007) 1005

13. Wang, W. PASP 120 (2008) 118

14. Wang, W. et al. A\&SA 496 (2009) 713

15. Woosley, S. E. in Saas-Fee Advanced Course 16: Nucleosynthesis and Chemical Evolution ed. Audouze, J. and Chiosi, C. and Woosley, S. E. (1986) 1

16. Woosley, S. E. and Weaver, T. A. ApJS 101 (1995) 181

17. Woosley, S. E. \& Heger, A. Physics Reports 442 (2007) 269 